\title{
The Role of Diagnostic Imaging in Macular Telangiectasia Type 2
}

\author{
Brett Zerbinopoulos, OD; Elina Goman-Baskin, OD; Paul B. Greenberg, MD, MPH; Richard Bryan, MD, PhD; \\ and Claire Messina, OD
}

While uncommon with subtle findings, macular telangiectasia type 2 can be diagnosed
with careful retinal examination and selective use of diagnostic imaging.

Author affiliations can be found at the end of the article.

Correspondence: Claire Messina

(cjcmessina@icloud.com)

Fed Pract. 2021;38(12). Published online December 13. doi:10.12788/fp.0200
$\mathrm{M}$ acular telangiectasia type 2 (MacTel2) is an uncommon, bilateral, and asymmetric condition that typically presents between the ages of 40 and 60 years without sex predilection. ${ }^{1-9}$ Its estimated prevalence ranges from 0.02 to $0.10 \%$. $^{2,8}$ The disease can manifest in either a nonproliferative or proliferative phase; the latter is far less common. The etiology of MacTel2 is poorly understood, but it is believed to have neurodegenerative as well as vascular components. ${ }^{1-6,8-10} \mathrm{We}$ present a case of MacTel2 and highlight the role of diagnostic imaging in early diagnosis prior to development of classic funduscopic features.

\section{CASE PRESENTATION}

A 66-year-old White male with a 10-year history of type 2 diabetes mellitus (T2DM) presented to the eye clinic for an annual eye examination. The patient was taking metformin, and 6 months prior to presentation, his hemoglobin $A_{1 c}$ was $7.4 \%$. He had a history of mild nonproliferative diabetic retinopathy in the left eye without diabetic macular edema. He reported no ocular concerns.

On examination, best-corrected visual acuity (VA) was 20/20 in each eye. Slit-lamp examination was notable only for bilateral mild nuclear sclerosis. Dilated fundus examination showed a blunted foveal reflex consistent with the appearance of a macular pseudo-hole in the right eye and was unremarkable in the left eye (Figure 1).

Macular optical coherence tomography (OCT) revealed an intraretinal cyst without thickening in the temporal fovea of both eyes with mild disruption of the underlying ellipsoid zone in the right eye (Figure 2). A presumptive diagnosis of MacTel2 vs diabetic

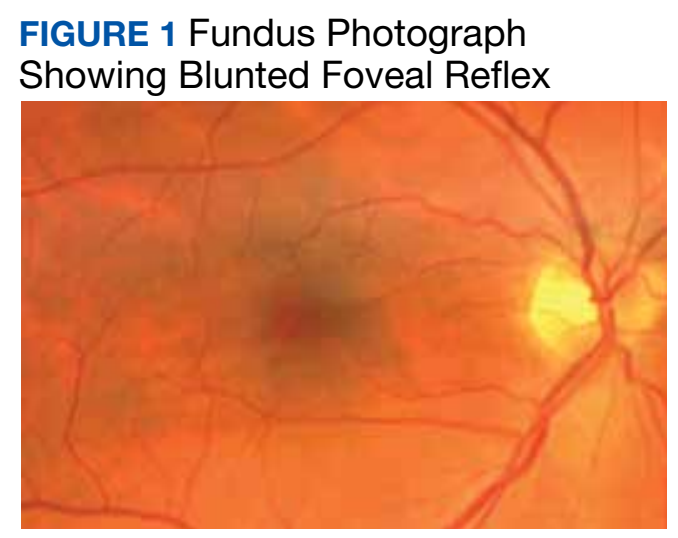

FIGURE 2 Macular Optical Coherence Tomography Showing Intraretinal Cyst

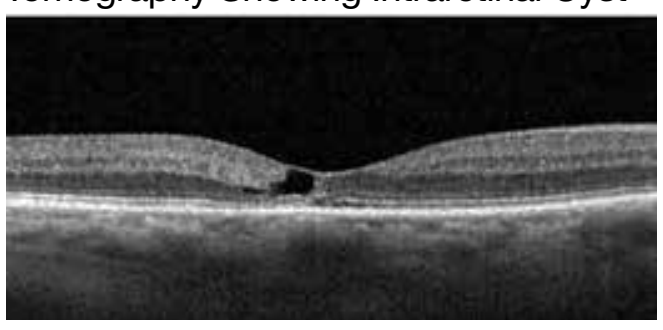

macular edema was made, and the patient was referred to the retina clinic for further evaluation.

At the 1-month follow-up in the retina clinic, VA, macula OCT, and fundus examination were stable. Fundus autofluorescence (FAF), optical coherence tomography angiography (OCT-A), and fluorescein angiography (FA) were performed. The FAF revealed a hyperreflective crescent in the temporal aspect of the fovea of both eyes, greater in the right eye than the left (Figure 3). The OCT-A showed abnormal dilation of the vessels in the deep capillary plexus of the temporal fovea of both eyes (Figure 4). This area of abnormality correlated to the area of 
hyperreflectivity seen on FAF. The earlyphase FA revealed telangiectatic vessels in the temporal fovea in both eyes; in the late phase, there was leakage of telangiectatic vessels, which remained localized to the temporal perifovea and spared the central fovea of both eyes (Figure 5). The patient was diagnosed with MacTel2.

\section{DISCUSSION}

This case highlights several important management considerations in MacTel2. These include symptoms, disease stage, and diagnostic imaging, which can allow more precise staging of the disease.

The etiology of MacTel2 is unknown. ${ }^{6}$ It is believed to be primarily a neurodegenerative condition that damages Müller cells and photoreceptors, leading to vascular changes..$^{1-6,8-10}$ Müller cells may play a role in creating and maintaining the integrity of the bloodretinal barrier, particularly in the deep capillary plexus where the vascular abnormalities begin. ${ }^{6,10}$ These early changes in the deep capillary plexus may evolve to include the superficial capillary plexus in intermediate stages with anastomoses forming between the 2 layers. ${ }^{2,6-10}$ Late proliferative stages show significant alterations of the juxtafoveal capillary network, subretinal neovascularization and retinochoroidal anastomoses. ${ }^{6,7,9,11}$ In one cohort study, $81 \%$ of patients with MacTel2 were White, and a genetic link is still under investigation. ${ }^{2,4-9}$

\section{Presentation}

The most common symptoms of MacTel2 include blurred vision, microscotoma, metamorphopsia, and difficulty reading, with missing or distorted letters a common concern. ${ }^{1,2,4-8}$ Best-corrected VA at presentation is usually better than $20 / 30$, and disease progression tends to be slow. ${ }^{2,6}$ Microscotomata are best mapped with microperimetry. ${ }^{1-3,5-7}$

There are several classic fundus findings (Table). In the early stages, these findings are subtle or entirely absent funduscopically. 1,2,4-10 In intermediate stages, fundus findings become apparent and include a loss of retinal clarity (grayish perifoveal sheen), telangiectatic macular vessels, retinal pigment epithelium hypertrophy, blunted right-angled vessels, and superficial retinal crystalline deposits., ${ }^{2,-11}$ Right-angled vessels

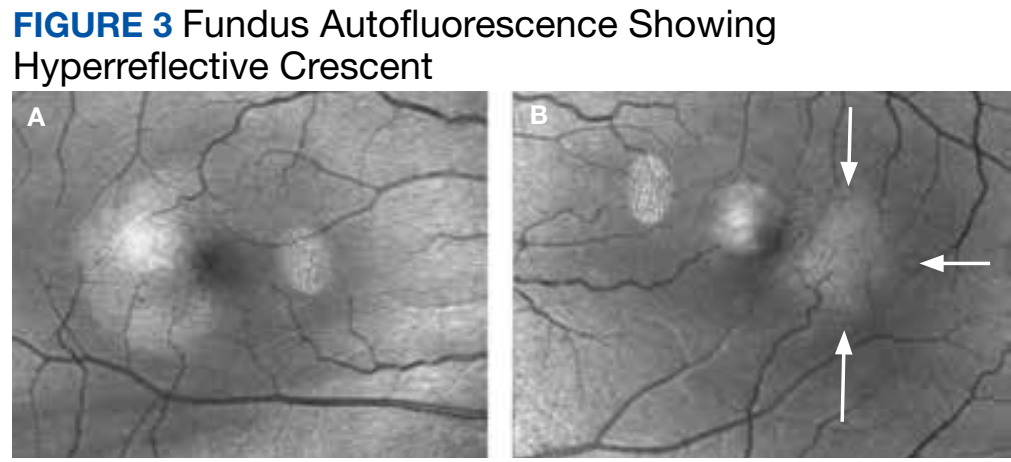

FIGURE 4 Optical Coherence Tomography Angiography Showing Abnormal Dilation

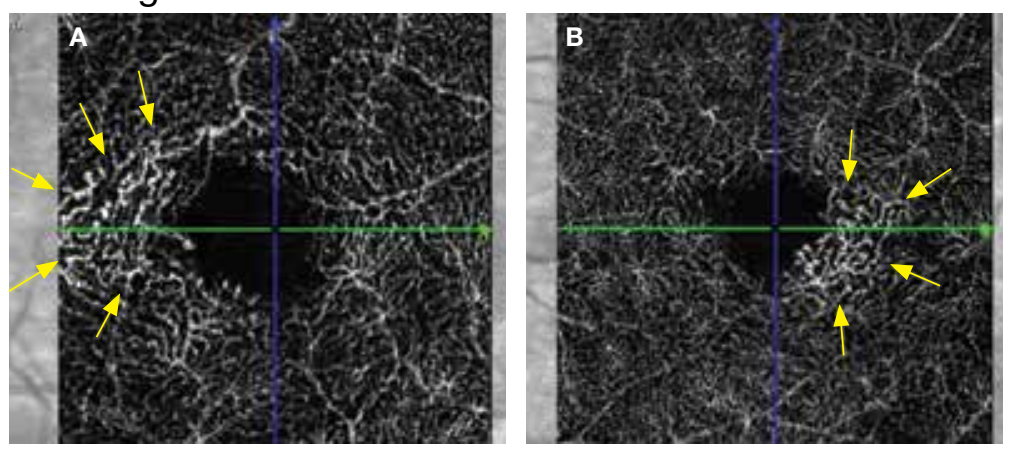

FIGURE 5 Fluorescein Angiography Showing Latephase Telangiectatic Vessel Leakage

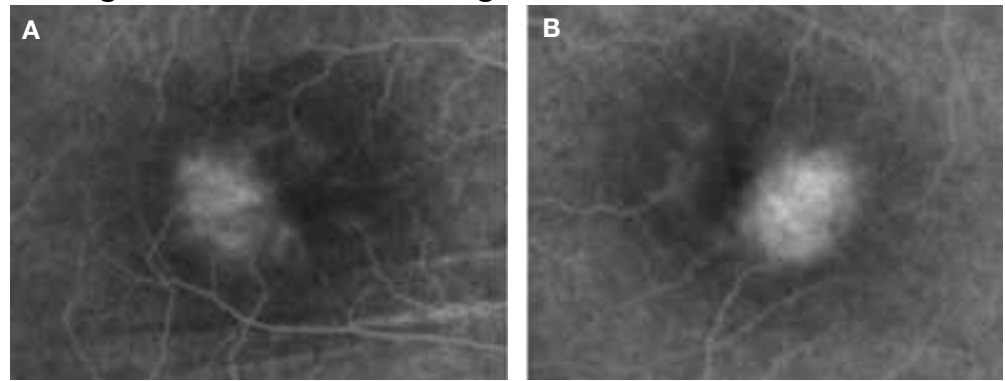

$A$, right eye; $B$, left eye.

may have a greater association with choroidal neovascularization, with growth into the outer retina in particular being a marker of disease progression. ${ }^{9}$ The crystalline deposits have been hypothesized to be the footplates of degenerated Müller cells. ${ }^{6}$

An important vision-threatening complication of MacTel2 is progression to proliferative disease. ${ }^{1,2,5-10}$ Choroidal neovascularization is present in a minority of cases and is associated with rapid vision loss. ${ }^{2,6} \mathrm{It}$ is often accompanied by subretinal hemorrhage and lipid exudation. ${ }^{6,7,9}$ If untreated, the result can be disciform scarring and fibrosis. ${ }^{2,5,6}$ Additional complications of MacTel2 are foveal atrophy and full thickness 
TABLE Fundus Findings in Macular Telangiectasia Type $2^{11}$

\begin{tabular}{lc} 
Fundus Findings & Prevalence, \% \\
\hline Loss of retinal clarity (grayish perifoveal sheen) & 74 \\
\hline Telangiectatic macular vessels & 51 \\
\hline Retinal pigment epithelium hypertrophy & 33 \\
\hline Blunted right-angled vessels & 29 \\
\hline Superficial retinal crystalline deposits & $21-46$ \\
\hline Choroidal neovascularization & $2-14$
\end{tabular}

macular holes. ${ }^{1,2,4-8,}$ Macular holes secondary to MacTel2 respond poorly to pars plana vitrectomy with inner limiting membrane (ILM) peel. ${ }^{2,6}$

\section{Diagnostic Testing}

Diagnostic retinal imaging is invaluable in the diagnosis of MacTel2. The OCT can detect hyporeflectivity within the ellipsoid zone in early disease corresponding to ellipsoid zone loss, which increases as the disease progresses. ${ }^{1-8,10}$ This loss most often begins in the temporal parafoveal region and correlates with the progression of both relative and absolute scotomas perceived by affected individuals. $2,3,5,8$

Intraretinal foveal hyporeflective spaces on the OCT represent cavity formation after Müller cell and photoreceptor loss and do not correlate with increased thickness. ${ }^{1,2,4,6,7}$ This is important in differentiating from diabetic macular edema, which will often show thickening. ${ }^{6}$ In most cases of MacTel2, foveal thickness is decreased. ${ }^{4-6}$ The ILM remains intact overlying this space and is referred to as ILM drape. , $^{6,7}$ This can cause blunting or absence of the foveal light reflex and mimic the appearance of a macular pseudohole. ${ }^{4}$

The OCT-A allows visualization of capillary changes through every layer of the retina, which could not otherwise be appreciated, allowing early detection as well as precise staging of the disease. ${ }^{2,6-10}$ Anastomoses present in late-stage disease also can be imaged using OCT-A. ${ }^{7,9}$ These anastomoses can be seen as hyperreflective vasculature present between the retinal layers where there is little to no vasculature visible in normal eyes. ${ }^{7}$

A lesser-known occurrence in MacTel2 is the depletion of macular luteal pigment, with many eyes possessing an abnormal distribution. ${ }^{2,4,6-8,10}$ This depletion and abnormal distribution can be visualized with FAF. In particular, short wavelength fundus autofluorescence (SW-FAF) is the most effective at highlighting these changes. ${ }^{10}$ The characteristic finding is a hyperreflective halo surrounding the fovea. ${ }^{2,6}$ Fluorescence life imaging ophthalmoscopy (FLIO) is a recent development in FAF that measures FAF lifetime, which is the duration of time a structure autofluoresces. ${ }^{8}$ A cross-sectional study published in 2018 showed prolonged FAF lifetime in the temporal fovea of patients with early and moderate stage MacTel2 when compared with normal patients. ${ }^{8}$ More advanced stages showed a ring encircling the entire fovea. ${ }^{8}$

Classic FA findings in MacTel2 include early hyperfluorescence of temporal foveal telangiectatic capillaries and late-stage leakage with sparing of the central fovea. ${ }^{1,2,4,6,7,11}$

\section{Management and Prognosis}

Management of MacTel2 depends on the stage of the disease. In the absence of proven treatment, management in nonproliferative stages is conservative. ${ }^{2,6}$ Intravitreal antiVEGF does not offer any benefit in nonproliferative disease. ${ }^{2,5,6}$ Indeed, as VEGF may have a neuroprotective effect on the retina, anti-VEGF may result in more harm than benefit in earlier disease stages. ${ }^{5}$ In proliferative stages, intravitreal anti-VEGF can help limit scarring and prevent vision loss. ${ }^{2,5}$

Long-term prognosis of MacTel2 is variable with VA typically better than 20/100. ${ }^{2}$ Vision loss in MacTel2 most often begins paracentrally; it can then progress centrally, leading to significant reduction in VA. ${ }^{12}$ The progression of this functional vision loss and corresponding structural damage is typically slow. ${ }^{3}$ VA worse than $20 / 100$ is usually a result of proliferative disease; in such cases, there is potential for severe central vision loss and legal blindness. ${ }^{1}$

\section{CONCLUSIONS}

This case of MacTel2 underscores the subthe retinal findings in the earliest stages of the disease and the importance of a complete retinal examination and diagnostic imaging with macula OCT, OCT-A, and FAF to establish the correct diagnosis. 


\section{Author affiliations}

Brett Zerbinopoulos is an Optometrist; Elina Goman-Baskin is an Optometrist; Paul Greenberg is an Ophthalmologist; Richard Bryan is an Ophthalmologist; and Claire Messina is an Optometrist; all at the Eye Clinic, Providence Veterans Affairs Medical Center in Rhode Island. Paul Greenberg is a Professor of Surgery and Richard Bryan is a Clinical Instructor of Surgery, both at the Division of Ophthalmology, Alpert Medical School, Brown University in Providence. Brett Zerbinopoulos is a Resident; Elina Goman-Baskin is a Clinical Instructor; and Claire Messina is a Clinical Instructor; all at New England College of Optometry in Boston, Massachusetts.

\section{Author disclosures}

The authors report no actual or potential conflicts of interest or outside sources of funding with regard to this article.

\section{Disclaimer}

The opinions expressed herein are those of the authors and do not necessarily reflect those of Federal Practitioner, Frontline Medical Communications Inc., the US Government, or any of its agencies. This article may discuss unlabeled or investigational use of certain drugs. Please review the complete prescribing information for specific drugs or drug combinations -including indications, contraindications, warnings, and adverse effects-before administering pharmacologic therapy to patients.

\section{Ethics and consent}

All authors have adhered to ethical principles for medical research. Informed consent was obtained from the subject involved in the study who was fully aware that a case study might be published. No identifying personal information is present.

\section{References}

1. Chew EY, Clemons TE, Jaffe GJ, et al. Effect of ciliary neurotrophic factor on retinal neurodegeneration in patients with macular telangiectasia type 2: a randomized clinical trial. Ophthalmology. 2019;126(4):540-549. doi:10.1016/j.ophtha.2018.09.041
2. Christakis PG, Fine HF, Wiley HE. The diagnosis and management of macular telangiectasia. Ophthalmic Surg Lasers Imaging Retina. 2019;50(3):139-144. doi:10.3928/23258160-20190301-02

3. Heeren TFC, Kitka D, Florea D, et al. Longitudinal correlation of ellipsoid zone loss and functional loss in macular telangiectasia type 2. Retina. 2018;38 Suppl 1(suppl 1):S20-S26. doi:10.1097/IAE.0000000000001715

4. Charbel Issa P, Heeren TF, Kupitz EH, Holz FG, Berendschot TT. Very early disease manifestations of macular telangiectasia type 2. Retina. 2016;36(3):524-534. doi:10.1097/IAE.0000000000000863

5. Khodabande A, Roohipoor R, Zamani J, et al. Management of idiopathic macular telangiectasia type 2. Ophthalmol Ther. 2019;8(2):155-175. doi:10.1007/s40123-019-0170-1

6. Wu L. When is macular edema not macular edema? An update on macular telangiectasia type 2. Taiwan J Ophthalmol. 2015;5(4):149-155. doi:10.1016/j.tjo.2015.09.001

7. Roisman L, Rosenfeld PJ. Optical Coherence Tomography Angiography of Macular Telangiectasia Type 2. Dev Ophthalmol. 2016;56:146-158. doi:10.1159/000442807

8. Sauer L, Gensure RH, Hammer M, Bernstein PS. Fluorescence lifetime imaging ophthalmoscopy: a novel way to assess macular telangiectasia type 2. Ophthalmol Retina. 2018;2(6):587-598. doi:10.1016/j.oret.2017.10.008

9. Tzaridis S, Heeren T, Mai C, et al. Right-angled vessels in macular telangiectasia type 2. Br J Ophthalmol. 2021;105(9):1289-1296. doi:10.1136/bjophthalmol-2018-313364

10. Micevych PS, Lee HE, Fawzi AA. Overlap between telangiectasia and photoreceptor loss increases with progression of macular telangiectasia type 2. PLoS One. 2019;14(10):e0224393. Published 2019 Oct 28. doi:10.1371/journal.pone.0224393

11. Gass JD, Oyakawa RT. Idiopathic juxtafoveolar retinal telangiectasis. Arch Ophthalmol. 1982;100(5):769-780. doi:10.1001/archopht.1982.01030030773010

12. Heeren TF, Clemons T, Scholl HP, Bird AC, Holz FG, Charbel Issa P. Progression of vision loss in macular telangiectasia type 2. Invest Ophthalmol Vis Sci. 2015;56(6):3905-3912. doi:10.1167/iovs.15-16915 